\title{
The progress of science from a computational point of view: the drive towards ever higher solvability
}

\author{
Witold Marciszewski*
}

\begin{abstract}
This essay's content is rendered by the titles of the successive sections. 1 . Effective solvability versus intuitive solvability. - 2. Decidability, i.e. effective solvability, in predicate logic. The speedup phenomenon - 3. Contributions of the secondorder logic to the problems of solvability - 4. The infinite progress of science in the light of Turing's idea of the oracle. The term "oracle" is a technical counterpart of the notion of mathematical intuition.

A more detailed summary can be obtained through juxtaposing the textboxes labelled with letters A...F. Conclusion: in the progress of science an essential role is played by the feedback between intellectual intuitions (intuitive solvability) and algorithmic procedures (effective solvability).
\end{abstract}

Keywords: effective solvability, intuitive solvability, decidability, speedup, secondorder logic, solvability, oracle, mathematical intuition, progress of science, algorithmic procedures.

Wir müssen wissen, wir werden wissen.

David Hilbert, the punch line of his lecture [1930]

The advance of science is not comparable to the changes of a city, where old edifices are torn down to give place to new, but to the continuous evolution of zoologic types which develop ceaselessly, where an expert eye finds always traces of the prior work of the centuries past.

Henri Poincaré, "The Value of Science", Introduction.

\section{The idea of effective solvability}

The author's mailing addresses: witmar@calculemus.org, witold@marciszewski.eu. 
1.1. The progress of science, characteristic of the Western civilization, embraces two processes: (i) the increase of the amount of methodically checked information, based on a reliable evidence, (ii) the development of more and more efficient methods and means of problem-solving via information processing.

The notion of science, as conceived in this essay, is pertinent to all academic disciplines in which a methodological research is carried on. Not only to empirical sciences, as it is frequently understood in the English literature. Instead, I use the term "science" rather in the sense of German "Wissenschaft".

The computational approach, as here understood, extends across at several areas. Not only computer science but also mathematical logic, some issues concerning the measure of information, considerations on the interaction between mechanical and non-mechanical (intuitive, creative) ways of problem-solving.

The choice of the term "solvability" in the title is premeditated to connect its meaning with the meanings of "decidability" and "intuition".

A. Effective solvability. A decision problem $\mathrm{P}$ is said to be decidable or effectively solvable when $\mathrm{P}$ can be solved by the universal Turing machine.

In the idiom of logic and computer science, effectiveness, or (another word) algorithmicity, is a specific difference (Lat. differentia specifica) to distinguish decidability from other solvability kinds; among them the key role is played by the intuitive solvability. We call so the cognitive power being the source of mathematical axioms, logical rules of inference, algorithms, and those innumerable understandings from which stem scientific concepts, as well as our everyday wisdom.

To obtain a figurative account of the relationship between algorithm and intuition, or invention, let us see the former as a tool, the latter as a human hand to use the tool.

Tools are as necessary for human civilization as are hands and brains, but they

would had never arisen without an inventive, typically human, insight.

This cognitive omnipresence of intuition makes it difficult to suggest an adequate definition. This may explain why some scholars are suspicious about this concept and tend to disqualify it as unscientific. However, there is no need to defend this concept by attempting a complete definition. Instead we have a cluster of partial operational definitions to be considered in sequel, and those belong to the core of logic and computer science, not unrespectable sciences. I mean such partial definitions as those being implicit in Gödel's [8] speeedup theorem and Turing's [27] logic defined by ordinals. They should exemplify what I mean using the word "intuition".

1.2. The above comment on this essay's title is meant as an introduction to the main subject of this essay. Let the second introducing comment pertain to the opening mottos. Both are highly relevant to the dynamics of scientific progress.

Far from such awareness are some fashionable currents in philosophy of science as fictionalism, conventionalism, postmodernism, etc. They eagerly oppose the rationalist optimism of Hilbert's maxim.

To understand this optimism, one needs some familiarity with the formalization of logic as presented in the textbook by Hilbert and Ackermann "Grundzüge der theoretischen Logik" [11], and in the two volumes by Hilbert and Bernays "Grundlagen der Mathematik" 
[13]. Formalization means that the procedure of checking the correctness of proofs is purely mechanical. No intuitive understanding of formulas is needed, it is enough to apply mechanical operations to the physical shapes of formulas. Such a formalization of a theory is a necessary (though not sufficient) prerequisite to algorithmization.

As for propositional logic, it was not only formalized but also algorithmized. This means that about any formula an algorithm could decide whether it is or is not a logical truth. As for predicate logic, no algorithm has been known, but up the thirties of the 20th century, its finding seemed to have been within reach. This common belief was eloquently expressed by the very then eminent philosopher of logic Ludwig Wittgenstein in his seminal book "Tractatus Logico-Philosophicus" (see items of [29]: 5.551, 6.125, 6.1251, 6.1262; quoted here after [14], p 729).

«Our fundamental principle is that every question which can be decided at all by logic can be decided off-hand. [...] It is possible [...] to give at the outset a description of all "true" logical propositions. Hence there have never be surprises in logic. [...] Proof in logic is only a mechanical expedient to facilitate the recognition of tautology.»

Were this statement right, then every mathematical problem would be solved algorithmically, hence tractable by a machine (note the term "mechanical expedient" in the quoted text).

Such was Hilbert's expectation in his saying "wir werden wissen". This expectation has not been fulfilled in its entirety, but even a partial fulfilment, as shown by Gödel does suffice to justify the rationalistic optimism pertaining to the progress of science. Hence that motto.

As for Poincaré's vision of scientific progress, it is close to that developed in this essay, in contradistinction to the opinions of those scientists or philosophers of science who maintained that there was to come the end of progress. Here are some anecdotic examples of that pessimistic finitism.

The physics professor Philipp von Jolly advised Max Planck against going into physics, saying, "in this field, almost everything is already discovered, and all that remains is to fill a few holes". The same view was entertained by Planck's friend Hermann von Helmholz. Ironically, it was Planck who soon initiated the conception of quanta, one of the most astonishing discoveries in the history of physics.

A vision also different from Poincaré's, though in another way, is represented in Thomas Kuhn's very influential book "The Structure of Scientific Revolutions" [15]. Revolutions are stormy events in which "old edifices are torn down to give place to new (as Poincare expressed the view that he opposed). The computational approach here adopted does not approve such an extreme view, when defining the progress of science as a constant increase of information and a drive towards ever greater solvability.

What Kuhn calls revolutions are, in fact, dramatic leaps forward. Such were, for instance, Cantor's set theory, Fregean logic, relativity, quanta, and so on. However, unlike political revolutions, they did not subvert the foundations and outputs of previous systems. Einstein's ideas about gravity didn't disprove but just enhanced those of Newton; nonEulidean geometries did not destruct the everlasting achievement of Euclid; the Fregean logic did not eliminate the Aristotelian contribution but has absorbed it as a constituent the monadic predicate calculus. 


\section{Decidability, i.e. effective solvability, in predicate logic. The speedup phenomenon}

2.1. As explained above in 1.1 , the term "decidability" is taken in this essay in the technical sense given to the German "Entscheidbarkeit", as understood by David Hilbert [1928, see 1.2] in the context of "Entscheidungsproblem" (see below 2.2). This property of a theory consists in the possibility of deciding in a way being mechanical or (in other words) algorithmic or else formal, whether the statement in question is true or false.

This meaning of "decidability" is narrower than in the ordinary English as explained in dictionaries: to decide - to reach a conclusion or form a judgement or opinion about something by reasoning or consideration. No mention is here done that decidability should be algorithmic. In that ordinary sense, the mathematical proofs - from those by Euclid to that by Andrew Wiles, pertaining to Fermat's theorem - though intuitive, not algorithmic, are unanimously regarded as leading to decisive conclusions.

To avoid misunderstandings in using the term "decidability", it is advisable in the present discussion to stick to its technical meaning (referring to an algorithmic procedure), and to customize another term to denote decidability in the broad ordinary sense. The candidate which seems the best is the word solvability as pre-announced in 1.1.

To decide a problem means to solve it in an algorithmic way; this means that deciding is a kind of problem-solving, apart of which there are other varieties of solving problems.

Among them, the most significant for intellectual progress is the intuitive problemsolving. Creative intuitions are at the roots of human knowledge to produce mathematical axioms, logical rules of inference, the concepts of class, number, entailment, computation, and so on. Even the algorithms themselves, as rules of mechanical problem-solving, owe their existence to non-mechanical inventive insights of the human mind. Kurt Gödel hinted at the axiom of mathematical induction, and the logical modus ponens rule, as typical and fundamental instances of such insights. One may still mention as equally fundamental the axiom of abstraction (referred to as AA below, 2.3 and 2.4).

The present author shares here the point which another author expressed as follows.

«Formal decidability is a concept relative to a given formalization of a mathematical

theory, and consequently, the fact that some sentence is undecidable in a formal theory does not give us any hint as to whether it is intuitively solvable» [21, page 47].

Let us have a look at the process in which intuition produces algorithms in order to give birth to new intuitions - as tools of the problem-solving at a new level. For instance, the intuition of Greek mathematicians gave birth to the algorithm of computing fields of triangles; this, in turn, gave birth to the intuition of irrational numbers. Such a feedback is what incessantly shifts the frontiers of science forward.

2.2. The story of decidability starts from Hilbert's [11] (see above 1.2) text which sounds like a more explicit version of his concise maxim quoted at the beginning. In this book Hilbert expresses the expectation that the first-order predicate logic, henceforth abbreviated as FOL, will prove to be decidable. This, in turn, would warrant the decidability of the whole of mathematics, since any mathematical formula can be recorded in the symbolism of predicate logic, and then mathematical proofs take advantage of its inference rules.

The text in question is found in Hilbert's and Ackerman's textbook [11, p. 73]. It reads as follows (ad hoc translation from German - by WM). 
B. Hilbert's Entscheidungsproblem. "The decision problem gets solved if one knows a procedure which for a given logical expression allows to decide, with finitely many steps, about its validity or its satisfiability. The solution of this decision problem has a fundamental impact for all those theories whose statements are capable of being logically derived from finitely many axioms."

This is the famous Entscheidungsproblem. It paved the way to Turing's crucial study that has laid the foundations of computer science - "On computable numbers with an application to the Entscheidungsproblem" [26]. Thus, were the predicate logic decidable, then the provability in mathematics would be trivial - in the sense of being dictated by an algorithm (as believed by Wittgenstein. cp. 1.2). That is, each mathematical problem could be solved by Turing machine.

It is worth noting that Hilbert in this famous statement was more heedful than Wittgenstein as quoted in 1.2. What the latter regarded as beyond any doubt, for Hilbert, the great master of logic, was seen as a problem to be studied. He admitted, not without a hope, the answer in the affirmative; however, he did not exclude the negative solution, and just the latter has turned out to be the case.

2.3. Before Turing proved in the whole generality the fact of FOL undecidability, Gödel [7] had demonstrated a special case that has an enormous bearings on the progress in mathematics. It is the famous theorem of the incompleteness of number theory (i.e., arithmetic of natural numbers) to the effect that there exist in it true propositions which are not provable from its axioms with the means of number theory and FOL.

The incompleteness entails the lack of decidability since it means that FOL does not provide such a universal algorithm which about any formula of arithmetic would decide whether it is provable from its axioms; let these axioms be labeled as A.

Algorithms warrant the finiteness of procedures. In this case it would be the procedure of deducing the formula in question, say Q, from A with the use of FOL inference rules. If one does succeed in deducing conclusion in this way, this indicates that the implication $\mathrm{A} \Rightarrow$ $\mathrm{Q}$ is necessarily true ${ }^{1}$.

Since the axioms A are assumed to be true, the consequent $\mathrm{Q}$ has to be true as well, and thus the problem gets decided. However, if after a fairly great number of successive steps the reasoner does not attain at conclusion, this leaves him in a state of uncertainty. For however long the time he has spent in systematic but unsuccessful search, a proof to bring decision may yet be found.

However, the state of knowledge in which it is impossible to derive some formula from the given axioms (say A) of a theory is not doomed to be immutable. Formal decidability, a property characteristic of Turing machines, is relative to a given formalization of a theory, that is to the set of axioms and inference rules of the logic having been adopted.

1 This is warranted by the deduction theorem (deemed most important in metalogic) to the effect that in order to prove the truth of an implication it is sufficient to derive the consequent (here Q) from the antecedent (here A). The history of this famous theorem goes back to a text by B. Bolzano of 1837, its proof is due to Alfred Tarski [25], and the name was given by Hilbert and Bernays [13]. For these and other details see [23], pp. 77-81. 
Consequently, the fact that some sentence is undecidable in a formalized theory does not give us any hint as to whether it is intuitively solvable ${ }^{2}$.

The fact that the range of decidability can to some degree be extended, either by reinforcement of axioms, or with enhancing methods of problem-solving (computing, proving, etc.), opens a new field of research - the search for such fertile axioms and methods.

Once more fact has been noticed due to such a search. The same new means which in the problem-solving procedure extend the range of decidability can cause this process to speed up, and thus cause a significant saving of such resources as working time and memory space. The first who made this observation and suggested some devices for such purposes was Kurt Gödel. Hence the choice of the name the Gödelian speedup to refer to this phenomenon.

In our times, this law of acceleration has an enormous impact on the progress of science. Such an awareness is mainly due to the computational point of view.

2.4. The term "speedup" is very popular in the computer science. When consulting Google about it, using as key words «speedup "computer science"», one obtains ca. 20 million answers. Some applications of this concept are concerned with computer architecture, other ones with software. Among the latter there are those algorithms, or programs, which should solve problems pertinent to Hilbert's Entscheidungsproblem.

Everybody knows that proofs which solve the same problem, that is, either prove or disprove a statement, may differ with their elegance, inventiveness, and, what counts here most, with the length, that is, the number of steps (lines) of inference. The bigger the length, the more time requires the process of proving. Consequently, any shortening of a proof results in accelerating its execution, hence this feature is referred to as speedup.

Under much telling title "Über die Länge von Beweisen" (On the length of proofs) Gödel [8] has made a pioneering step towards the branch of computer science which nowadays is called computational (or algorithmic) complexity theory (see https://plato.stanford.edu/entries/computational-complexity/). His seminal statement reads as follows.

C. Gödelian speedup. "Thus, passing to the logic of the new higher order has the effect, not only of (1) making provable certain propositions which were not provable before, but also of (2) making it possible to shorten, by an extraordinary amount, infinitely many of the proofs already available." (English translation 1986, p. 397; numbers added by WM.)

"Already available" means those obtained at a lower order of logic ${ }^{3}$.

2 Formalization of a theory means that the logical correctness of any proof can be checked by mechanical procedure. It consists in a sequence of operations transforming the physical forms of expressions into other physicals form; such operations and their succession are dictated by inference rules as a kind of algorithm. The term "proof" in the context of this discussion always denotes a proof formalized, i.e., mechanized, in such a way.

3 The first proof of this Gödel' theorem was given in [4]. See https://projecteuclid.org/euclid.jsl/1183744546. It is in order to notice that in the wikipedia 
The resort to higher-order logics requires a philosophical comment. It should be noted that such a step entails a deeper ontological involvement than that entailed by the first order logic. Thus the gain in time is obtained, allegedly, at the cost of a higher philosophical risk. The nature of such a risk can be read off from the following set-theoretical axiom. It has several names to hint at various aspects of its role. In the present context, the most suitable name will be "Axiom of Abstraction".

This axiom has two versions. Let AA be the label of the ZFC version in which a proviso is included to safeguard this statement against antinomies. The other version, to be here labelled as AA", appeared in Cantor's pre-axiomatic "naive" theory, i.e., without an awareness of destructive antinomies. This threat appears in a wider theoretical framework which does not occur in the present context, hence we disregard AA in it, and avail ourselves with a simpler and more transparent form, to wit $\mathrm{AA}^{\prime \prime}{ }^{4}$.

$$
\text { AA: } \forall \mathrm{z} \exists y \forall x(x \in y \Leftrightarrow x \in z \wedge \varphi(x))
$$

$$
\mathbf{A A}^{\prime \prime}: \exists \mathrm{y} \forall \mathrm{x}(\mathrm{x} \in \mathrm{y} \Leftrightarrow \varphi(\mathrm{x}))
$$

AA" is a theoretical basis to introduce the very convenient concept of abstraction operator. It transforms a sentential formula into a name of the class of those things which satisfy that formula. Let the formula be $\mathrm{Q}(\mathrm{x})$. The name in question is written as $\{\mathrm{x}: \mathrm{Q}(\mathrm{x})\}$. For example, $\mathrm{z} \in\{\mathrm{x}: \mathrm{x}<3\}$ means that $\mathrm{z}$ (interpreted as a natural number) is one of the numbers $0,1,2$.

Braces \{\} are used in set theory to designate a class (i.e., set); for instance $\{1,2,3\}$ is the class of those numbers which are listed inside. The combination of symbols forming this operator consists of such braces and the colon that follows the variable(s) being the argument(s) of the predicate preceded by the colon (the ending "s" hints at predicates having two or more arguments).

The abstraction operator is being introduced by definitions like the following.

$$
\begin{gathered}
\text { df.1) } z \in\{x: Q(x)\} \Leftrightarrow Q(z) \\
\text { df.2) }\langle\mathrm{t}, \mathrm{z}>\in\{\mathrm{x}, \mathrm{y}: \mathrm{R}(\mathrm{x}, \mathrm{y})\} \Leftrightarrow \mathrm{R}(\mathrm{t}, \mathrm{z})
\end{gathered}
$$

etc. for predicates of two arguments; the same pattern is adopted for predicates of arbitrary many arguments.

The operator of abstraction conveniently simplifies utterances concerning sets and relations. E.g., the universal set can be defined as $\{\mathrm{x}: \mathrm{x}=\mathrm{x})\}$, and the empty set as $\{\mathrm{x}: \mathrm{x} \neq \mathrm{x}\}$. Furthermore, other operators, e.g. quantifiers, can be introduced with the help of this operator.

From a philosophical point of view the abstraction operator is seen as a device to form

article "Gödel's speed-up theorem" there is erroneously said that this theorem was proved by Gödel. It is not true and hardly possible since the text in question is very short (only one page).

4 ZFC means the set-theoretical system of Zermelo and Fraenkel with added the axiom of Choice. In its practice (unlike in other systems), followed in present text, the term "class" means nothing different from "set", and is applied interchangeably for the sake of a stylistic convenience. See [6], p. 59, footnote 1. 
the name of an abstract entity (e.g., empty set, etc.) out of a predicate or a sentential formula. Let abstract entities be briefly called abstracts; they are denoted by abstract concepts.

The progress of mathematics and other disciplines is made by proving new theorems, and this, in turn, is due to inventing ever more abstract concepts. Such a creative activity lies at the roots of scientific advances.

This fact gives rise to a philosophical question: should we dare an ever deeper ontological commitment? A mathematical realist (usually called platonist) who believes not only in the existence of individuals, but also of classes, is deeper ontologically committed than a nominalist who allows only such existential statements which refer to individuals. If someone acknowledges the existence of sets of classes, hence even more abstract entities, becomes still deeper ontologically involved, and so on. There are philosophers, e.g. nominalists, who do not admit the existence of abstract entities, and refuse to accept mathematical results obtained under such assumption. However, who seriously wishes to do science should give up such scruples; he may be a nominalist in his personal philosophical conscience but as an active researcher in any field of science, he cannot do without abstract concepts, and so, in practice, he is bound to assume the existence of abstract entities.

2.5. Logical investigations concerning both the decision problem, as well as the metalogical properties of number theory, start usually from Peano Arithmetic (PA) as a reference point for both issues. The interest is due to the fact that about PA Gödel has proved his famous and seminal theorem of incompleteness and undecidability. This has incited the search for systems of arithmetic which would be complete and decidable.

Among such systems, the one that turned out to be much interesting and instructive from a computational point of view is Presburger arithmetic. It is the first-order theory of the natural numbers with addition, named in honour of the Polish Jewish logician, a student of Alfred Tarski, Mojżesz Presburger, who introduced it in 1929. The sole primitive concepts in its axioms are addition and equality, without multiplication. Among axioms there is the schema of abstraction in terms of first-order logic. So weakened systems proves to be consistent, complete and decidable, but at the cost of being unable (unlike PA) to formalize the concepts of multiplication, divisibility, prime number, etc.

Among the axioms of PA, a distinguished place is held by the axiom of induction in two versions: either the first-order or the second-order formulation. In the former case, its more exact name is "the axiom schema of induction" since it provides a schematic form for infinitely many concrete axioms.

The induction axiom in the first-order schematic form reads as follows ("Sx" means "the sequent of $x ")$.

$$
\text { 1st.AI: }[\varphi(0) \wedge \forall \mathrm{x}(\varphi(\mathrm{x}) \Rightarrow \varphi(\mathrm{Sx}))] \Rightarrow \forall \mathrm{x} \varphi(\mathrm{x})
$$

The second order form (2nd.AI) differs from 1st.AI in such a way that the whole formula is prefixed by the universal quantifier which binds a variable $\varphi$ to be conceived as ranging over properties or classes, hence abstract entities. Thus we obtain the following formula.

$$
\text { 2nd.AI: } \forall \varphi\{[\varphi(0) \wedge \forall \mathrm{x}(\varphi(\mathrm{x}) \Rightarrow \varphi(\mathrm{Sx}))] \Rightarrow \forall \mathrm{x} \varphi(\mathrm{x})\}
$$


The assertion of 2nd.AI entails the ontological commitment into the existence of classes or properties (in the above formula the property $\varphi$ defines a class - according to the axiom AA").

\section{Contributions of the second-order logic to the problems of solvability and decidability}

3.1. This section continues the subject of "Gödelian speedup" discussed in 2.4 and 2.5. Here the emphasis is to be put on the mechanical aspect of decidability, the closest to the computational perspective by the fact that "decidable" in the technical sense means decidable by Turing machine. From such a computational point of view let us reconsider the subject, starting from the summary of what has been said so far.

When we encounter a sentence, say $\mathrm{S}$, which is not decidable in the existing formalism, we can enlarge our formalism by adding some new apparatus, and so prove S. This new apparatus is more powerful inferentially, what can be obtained with the use of axioms containing variables of higher orders than those occurring in the system hitherto held. This requires the predicate logic in which quantified variables are of the same order as those occurring in the new axioms.

Gödel [8] has stated that such a passing to a higher order enables us to prove formulae that were hitherto unprovable and, moreover, allows very much shorter proofs for many of the previously obtainable formulae. Within this new system it is possible to construct a new undecidable formula, and so the whole process can be repeated as many times as is needed to obtain a desirable level of decidability, potentially in infinitum ${ }^{5}$.

The second part of Gödel's assertion, that concerning the shortening of proofs already available, not at once was duly appreciated. Only with the rise of the theory of computational complexity of algorithms people started to realize the significance of this part of Gödel's statement ${ }^{6}$.

The greater was becoming the computational power of hardware (increasing exponentially according to Moore's law), the more was growing the awareness of the significance of the computational power of software. Knowing both, the available speed of processors, and effectiveness of algorithms, we have grounds to estimate how complex and hard problems are we able to solve with such devices. In such a situation, Gödel's, as well as Turing's (see 4), message runs as follows. There are problems so hard that even most speedy computers, programmed with the fastest available algorithms, do not give us a hope of solving them even in some millions or more years. Such a bad news may be accompanied by a good one: that the state of devices needed to solve the problem in question is never definitive. New devices can be found owing to what Turing called solutions with "oracle" (see 4.2).

Practically, arguments to support the above contention focus on the enormous difference in computational power between the first-order and second-order logic. To obtain at least a rough idea of that difference, we can consider an example of reasoning in

5 This Gödel's intuitively grasped theorem has been formally proved by Buss, as mentioned in the footnote 2 .

6 This became the case twenty years later, esp. in the study [10]. 
that part of ordinary English which corresponds to some part of second-order logic.

In a village there are three parental couples, each having two little children, and there are no more parental couples in that village. Hence there are exactly six little children in that village.

Even in such a childish problem, one resorts to the second-order ideas when talking of sets (couples) as something existing. If a radical nominalist like, for example, Tadeusz Kotarbiński regards second-order logic as an unscientific metaphysics, he should do his best to express such a reasoning in the first-order language.

He would be bound to use in it six individual variables to speak of children $(\mathrm{x} 1, \ldots, \mathrm{x} 6)$ in order to state about any individual the fact of being a little child, and then to say about any other individual denoted by a variable, say y, that if y were a little child, then it would be identical either with $\mathrm{x} 1$ or $[\ldots]$ with $\mathrm{x} 6$. This would require using more than hundred symbols. The formalism needed to express the rest of this reasoning would be even more cumbersome, as involving binary relation of being, so to speak, parentally coupled, and ternary relation of being a child of a couple in question.

The comparison of the above first-order reasoning and that given above, in the English ordinary language of second order, testifies that some mental mechanisms of human reasoning spontaneously function according to the second-order pattern. Is it possible for computers to simulate and match humans also in this respect? This is a question to be addressed to those who blame the use of any higher-order language and try to design the first-order artificial intelligence restricted to the first-order logical apparatus.

More elaborate argument for indispensability of the higher-order means of expression has been presented in a very seminal paper by George Boolos [3]. Let the inference in question be called BP (for Boolos' Proof). I give up presenting the content of BP itself, and just follow a comment of Boolos, to hint at the size of difference between the lengths of first-order and second-order proofs. The latter, as shown by Boolos, needs the space of about one page, while the amount of symbols needed in the FOL derivation is represented by the exponential stack of as many 2's as 64536 . This is a number larger than any integer that might appear in science.

A significant source of this difference amounts to the fact that BP gets reduced to a very short derivation due to the use of abstraction axioms (hence the second-order formulas). They are propositions subsumed under the axiom schema of abstraction discussed in 2.4 (let us recall its content).

$$
\mathbf{A A}^{\prime \prime}: \exists \mathrm{y} \forall \mathrm{x}(\mathrm{x} \in \mathrm{y} \Leftrightarrow \varphi(\mathrm{x}))
$$

3.2. This fact is crucial for the next step of the inquiry into the feedback between creative human minds and machines. Let us consider the realm of mechanized deduction. It is the field in which researchers detect experimentally the machine's inability to create new abstract concepts, though necessary for the proof in question; what means inability to make use of the axioms of abstraction.

A thought-provoking report pertaining the deep difference between creative and mechanical problem-solving is due to Christoph Benzmüller and Manfred Kerber, renowned researchers in field of automated reasoning. Their survey of problems in that area is worth a literal quote $[1,2]$.

«Automating proof search in higher-order logic is a very challenging enterprise, such that the above systems all provide facilities to combine interaction with 
automation. The idea is that the interactive human provides the crucial proof steps while simple subgoals are handled automatically by the prover. Of course, many non-trivial proofs can be already automated in higher-order logic. [...] A well known example illustrating the expressiveness and elegance of automated higher order theorem proving is Cantor's theorem, where the diagonalisation argument, in form of a lambda-term, is synthesised by higher-order unification. However, Boolos' example perspicuously demonstrates the limitations of current first-order and higherorder theorem proving technology. With current technology it is not possible to find his proof automatically, even worse, automation seems very far out of reach. Let's first give a high-level description why this is so. Firstly, Boolos' proofs need comprehension principles [in this essay termed "axioms of abstraction"] to be available and it employs different complex instances

of them. [...] Secondly, the particular instances of the comprehension axioms cannot be determined by higher-order unification but are so-called Heurika-steps which have to be guessed. However, the required instantiations here are so complex that it is unrealistic to assume that they can be guessed. [...] Here it is where human intuition and creativity comes into play, and the question arises how this kind of creativity can be realised and mirrored in a theorem prover. »"

Let us connect the last sentences to to sum up the awareness of the most experienced inquirers of mechanized reasoning.

D. An opinion of experts on mechanized reasoning. "In the guessing of the most complex instantiations of the schema of comprehension (abstraction) axiom, that is, the most involved concepts, human intuition and creativity comes into play, and the question arises how this kind of creativity can be realised and mirrored in a [mechanical] theorem prover.»

The question raised in the last sentence is fundamental. A great challenge consists in simulating the process of concept-forming involved in the axiom of abstraction. It is specially perspicuous in Boolos' inference in which so great a role is played by searching for relevant substitutions of the schema of the abstraction axioms. Selecting useful axioms is the same question (the authors notice) as to form needed concepts.

3.3. All that is highly interesting and amenable for the discourse on the development of sciences, not only of mathematics. Mechanized deduction is essentially involved in knowledge management and expert systems, as well as artificial society projects, the latter using the computer simulation of social phenomena (with cellular automata, agent programs, etc.). All the listed enterprises, are crucial for the progress of social sciences. The input of mechanized deduction is feasible in them, since each of the mentioned tasks includes reasonings.

Let us have a look, like in a case study, into the problem-solving in economics in which (more than in other social sciences) the awareness of the following question has arisen: how wide in the economical matters is the range of solvability and decidability? Such awareness appeared in economics in the famous Hayek-Lange debate on socialist calculation (an account of that debate is given in [18]).

It was Oskar Lange who claimed all economic problems be solvable in the system of 
socialist central planning. It was Friedrich Hayek who claimed the computational intractability of central planning problems, and advised therefore to resort to free market mechanisms as using better methods of data-processing. To wit, the allowing that the market agents make decisions according to their own knowledge, is most computationally efficient and most advantageous, since the market acts as a computational device in the mode of parallel and interactive information processing.

However, what would be specially interesting in that debate, has not been explicitly identified by the adversaries. It is the problem of the kind of logic as used by market agents. Let me direct our attention to a deep observation in the paper by Boolos [3], p. 380. It runs as follows.

«The fact that we so readily recognize the validity of I [this label denotes Boolos famous inference] would seem to provide as strong a proof as could be asked for that no standard first-order logical system can be taken to be a satisfactory idealization of the psychological mechanisms or processes, whatever they might be, whereby we recognize logical consequences.»

Thus it does not seem unlikely that free market agents in their reasonings use higherorder logics without any computer assistance, so taking advantage of the Gödelian speed-up in their intuitive reasonings. On the other hand, a socialist planner (unlike a free market agent dealing with a reasonably restricted set of data) has to deal with so astronomically huge data amount that he is helpless without computer's assistance. However, that assistant is often doomed to fail in solving some problems at the second-order level - for the reasons discussed by Benzmüller and Kerber in their discussion of conceptual difficulties (as reported above, 3.2).

Now, leaving aside the above case study, let us look more generally at the growing interaction, in the form of positive feedback, between the development of mathematics and the increase of computational power. That increase, apart from being due to ever greater hardware perfection, heavily draws on software perfectioning. It is in order here to recall the lesson due to Gödel, Turing, Boolos and such researchers as Benzmüller, Kerber, Brown, etc. ${ }^{7}$.

\section{The infinite progress of science in the light of Turing's idea of the oracle}

4.1. The phrase "in the light" fittingly renders the role of the notion of oracle. To wit, its role for understanding the nature and perspectives of scientific progress. This notion, indeed, proves illuminating.

Being much absorbed in exploring Turing's most seminal study on computable numbers and decision problem [26], computer scientists and philosophers of mind long overlooked the idea of oracle which appeared in Turing's dissertation [27]. It has been recalled by Andrew Hoges in his biographical studies on Turing, including the extensive article "Alan Turing" in "Stanford Encyclopedia of Philosophy" (section 4 "The uncomputable". See https://plato.stanford.edu/entries/turing $)^{8}$.

Turing, being aware that intuition is present in every part of a mathematician's thought,

7 The present section 3 is a revised version of part the paper [19], on line: http://logika.uwb.edu.pl/studies/index.php?page=search\&vol=22

8 Section 4 "The uncomputable". See https://plato.stanford.edu/entries/turing. 
posed the question: what would result from supplementing a formal system (say, Turing machine) with intuitive uncomputable steps? To deal with this problem, he introduced the concept of an oracle - a device which should be able to answer the halting problem. He defined oracle as an uncountable function, and added the comment:

«We shall not go any further into the nature of this oracle apart from saying that it cannot be a machine.»

When connecting the oracle with intuition, Turing must have been convinced that it consists in seeing the truth of a formally unprovable proposition, like that detected by Gödel. It was also Gödel who discovered something like stairs leading towards ever higher degrees of decidability, according to growing numbering of the orders of logic (see above, 2.4 , textbox $\mathrm{C}$ and accompanying comments).

Turing generalized Gödel's vision with the following crucial statement opening his study.

E. Turing's logical stairs up to the infinity. The well known «theorem of Gödel shows that every system of logic is in a certain sense incomplete, but at the same time it indicates means whereby from a system $\mathrm{L}$ of logic a more complete system $\mathrm{L}^{*}$ may be obtained. By repeating the process we get a sequence $\mathrm{L}, \mathrm{L} 1=\mathrm{L} * \mathrm{~L} 2=$ $\mathrm{L} 1 *, \mathrm{~L} 3=\mathrm{L} 2 *, \ldots$ of logics each more complete than the preceding. A logic L $\omega$ may then be constructed in which the provable theorems are the totality of theorems provable with the help of the logics L, L1, L2,..., [...]»

This statement is a bridge between Gödel's and Turing's achievements, leading to an astonishing picture of infinite progress of science, according to Hilbert's brave maxim: wir werden wissen. Gödel and Turing make us aware of this dynamic phenomenon: the unending process of science's becoming, advancing its frontiers without any limit. This picture pertains to mathematics. However, when seeing mathematics as the leading force of scientific progress, we can generalise about a vast field of whole science. How it can be applied to a particular science, it is exemplified in 3.3 with the case of economics.

Turing mentions at the very start tha Gödel indicated means to successively obtain systems ever closer to complete, in which all true (in the given language) sentences can be proved. However, completeness does not necessarily entail decidability.

This has been commonly known in the case of first-order logic, after Gödel proved its completeness and Turing (as well as Church) proved its undecidability. On the other hand, completeness is necessary for decidability. Provided the completeness, the decidability can be obtained after doing an additional work, to wit: finding out an algorithmic procedure to prove or disprove any formula of the system in question. This can be achieved, and often does, owing to a suitable input of invention and diligence.

Thus, attaining to a theory $\mathrm{T}^{+}$more complete than a previous theory $\mathrm{T}$, is a step in the advance of science. It is a value in itself, and creates a chance of winning another value, that of decidability.

4.2. After having done (at the beginning of this section) some introductory remarks on the oracle, let us now consider the thing once more - in the light of the above Turing's utterance quoted in textbox E. Some pages later Turing completes that statement with the 
following idea.

F. Turing's idea of oracle. «Let us suppose that we are supplied with some unspecified means of solving number theoretic problems; a kind of oracle as it were. We will not go any further into the nature of this oracle than to say that it cannot be a machine; with the help of the oracle we could form a new kind of machine (call them o-machines), having as one of its fundamental processes that of solving a given number theoretic problem.» - "Systems of logic...", p. 13.

Let us pay attention to the adjective "unspecified". It evidences a sober well-balanced approach. Turing does not pretend to know the nature of this factor; this might be a subject of an inventive hypothesis, but Turing himself prefers to focus on an unquestionable fact, even if enigmatic. As unquestionable he saw that the oracle could not be a machine. Thus, having displayed a due consideration and reserve as to the nature of that enigmatic factor, Turing [26] accompanied Gödel in opposing the mechanistic trend ${ }^{9}$.

Though Turing himself did not explain explicitly his idea of oracle in terms or mental or physical processes, there are reasons to suppose that it was his intention to interpret "oracle" in terms of human intellectual capacity. On this interpretation, the oracle is related to the intuition capable of seeing the truth of a Gödel statement. M. H. A. Newman, who introduced Turing to mathematical logic and collaborated with him, wrote that the oracle resembles a mathematician "having an idea", as opposed to applying a mechanical method (cp. Andrew Hodges' article "Alan Turing" in: "Stanford Encyclopedia of Philosophy").

Thus it is certain that when working on logical systems based on ordinals, Turing was vividly interested in uncomputable operations in their relation to the mental intuition of truths, namely those which cannot be established by executing mechanical processes. How much the intellectual intuition does matter can be seen with reports of eminent experts in mechanized reasoning whose opinions are discussed above in 3.2.

\section{Conclusion}

To sum up this discussion, let us connect its main foci, to wit, those distinguished with the following framed texts.
A. Effective solvability
B. Hilbert's Entscheidungsproblem
C. Gödelian speedup
D. Opinion of experts on mechanized reasoning
E. Turing's logical stairs
F. Turing's idea of oracle.

Gödel's and Turing's reactions to Hilbert's problem gave a new reasonable sense to Hilbert's maxim Wir müssen wissen, wir werden wissen, in which "to know" was originally to mean: to have a problem solved through a formal (algorithmic, mechanical) proof, while an

9 An eloquent representative of mechanism is [28]. As for Turing, in his post-war period he did not express an reserve against mechanism, but also did not display any retreat from the ideas and results of his study [27]. This question is debated by commentators as a bit enigmatic. 
intuitive solution was not taken into account.

Gödel has demonstrated that an algorithmic decision is always thinkable, even if in a very distant time. However, it needs creative insights to obtain axioms and rules of a formal system as a prerequisite for new algorithms to meet new challenges. New algorithms not only widen the range of decidable questions but also make such a process much faster. The indispensable in all that role of human creative intuition has convincingly been illustrated by the quoted experts in mechanized reasoning (see textbox D).

Turing devised the logical framework in which for every machine able to (mechanically) decide problems from a certain set, there exists (i.e., can be produced) a more powerful machine able to decide any problem decidable by the previous one machine. Such an ascent ("stairs") up to ever stronger machines requires (at each transitory phase) a factor which Turing called "oracle", and understood it as mathematical intuition.

Thus, through the multiple iterating cycles of intuitive solutions and algorithmic (mechanical) decisions, there proceeds the gigantic progress of science towards an ideal state, dawning somewhere at the horizon of infinity ${ }^{10}$.

\section{References}

[1] Benzmüller Ch., Kerber M., A Challenge for Mechanized Deduction (to find the full text in Web, ask Google for the title and select the relevant PDF), 2001.

[2] Benzmueller Ch., Brown Ch., The Curious Inference of Boolos in Mizar and OMEGA, in: Studies in Logic, Grammar and Rhetoric (http://logika.uwb.edu.pl/studies/index.php?page=search\&vol=23) 23, 2007.

[3] Boolos G., A Curious Inference?, Journal of Philosophical Logic, 16, 1987, 1-12.

[4] Buss S.R., On Godel's Theorems on Lengths of Proofs I: Number of Lines and Speedup for Arithmetics, J. Symbolic Logic, 59, 3, 1994, 737-756,.

[5] Fischer M.J., Rabin M.O., Super-Exponential Complexity of Presburger Arithmetic, Proceedings of the SIAM-AMS Symposium in Applied Mathematics, 7, 1974, 27-41.

[6] Fraenkel A.A., Abstract Set Theory, North Holland, 1976.

[7] Gödel K., Über formal unentscheidbare Sätze der Principia Mathematica und verwandter Systeme I, Monatshefte für Mathematik und Physik, 38, 1931, 173198.

[8] Gödel K., Über die Lange von Beweisen, Ergeb. Math. Kolloquiums, 7, 1936, 23 24.

[9] Gödel K., Kurt Gödel Collected Works, vol. 1. Oxford Univ. Press, Oxford, 1986.

[10] Hartmanis J., Stearns R., On the computational complexity of algorithms, Transactions of the AMS, 117, 1965, 285-306.

[11] Hilbert D., Ackermann W., Grundzüge der theoretischen Logik, Springer, 1928.

[12] Hilbert D., Naturerkennen und Logik, Naturwissenschaften, Heft 47/48/49, 28.II.1930, 959-963.

[13] Hilbert D., Bernays P., Grundlagen der Mathematik, Springer, (vol. 1) 1934, (vol. 2) 1939.

[14] Kneale W., Kneale M., The Development of Logic, Clarendon Press, 1962.

10 A wider perspective on the issues discussed in this study can be found in the book [17]. 
[15] Kuhn T., The Structure of Scientific Revolutions, University of Chicago Press, 1962, rev. ed. 1970.

[16] Marciszewski W. (ed.), Dictionary of Logic as Applied in the Study of Language. Concepts, Methods, Theories, Nijhoff, 1981.

[17] Marciszewski W., Murawski R., Mechanization of Reasoning in a Historical Perspective, Rodopi, 1995.

[18] Marciszewski W., Hypercomputational vs. computational complexity. A challenge for methodology of the social sciences, in: Free Market and Computational Complexity. Essays in Commemoration of Friedrich Hayek (1899-1992) of the series Studies in Logic, Grammar and Rhetoric (http://logika.uwb.edu.pl/studies/index.php?page=search\&vol=18), 5(18), 2002.

[19] Marciszewski W., The Gödelian Speed-up and Other Strategies to Address Decidability and Tractability, Studies in Logic, Grammar and Rhetoric, 9(22), 2006.

[20] Newman M. H. A., Alan Mathison Turing, Biographical memoirs of the Royal Society, 1955, 253-263.

[21]Placek T., Mathematical Intuitionism and Intersubjectivity: A Critical Exposition of Arguments for Intuitionism, Springer Science \& Business Media, 1999.

[22] Poincaré H., The Value of Science (French La Valeur de la Science, 1905), Dover Publications, 1958.

[23] Surma S. J., Deduction theorem, in: Marciszewski (ed.), Dictionary of Logic as Applied in the Study of Language. Concepts, Methods, Theories, Nijhoff, 1981, 77-81.

[24] Tarski A., Logic, Semantics, Metamathematics, translated by J. H. Woodger, Clarendon Press, 1956.

[25] Tarski A., On some fundamental concepts of metamathematics, in: Tarski A., Logic, Semantics, Metamathematics, 1956, German original published in the proceedings of the Scientific Society of Warsaw, 1930.

[26] Turing A., On computable numbers with an application to the Entscheidungsproblem, Proc. of the London Math. Society, Series 2, 1936, 230265.

[27] Turing A., Systems of logic defined by ordinals, Proc. Lond. Math. Soc., Ser. 2, 45, 1939, 161-228.

[28] Webb J.C., Mechanism, Mentalism, and Metamathematics, Reidel, 1980.

[29] Wittgensten L., Tractatus Logico-Philosophicus, Routledge \& Kegan Paul, 1921.

Received 13.08.2018, Accepted 25.02.2019 